\title{
Supranuclear Horizontal Gaze Palsy Following Anterior Internal Capsule Hemorrhage
}

\section{Nathan Chu (D), Grayson Beecher (D), Mohammed Wasif Hussain}

Keywords: Neuroanatomy, hemorrhage - cerebral, neurological examination, neuro-ophthalmology, internal capsule, supranuclear, gaze palsy

An 86-year-old man with hypertension, insulin-dependent type 2 diabetes, and coronary artery disease on low-dose aspirin presented with acute onset right upper motor neuron facial weakness, right hemiparesis, and left gaze deviation. Ophthalmological examination demonstrated impaired rightward smooth pursuit and saccadic eye movements with preserved oculocephalic reflex in keeping with a supranuclear right horizontal gaze palsy (Figure 1A-D). There was no compensatory head turn.
Neuroimaging demonstrated a sole hemorrhage in the left posterior limb of the internal capsule with extension into the anterior internal capsule (Figure 1E-H). The patient was treated conservatively with blood pressure management and cessation of aspirin. His gaze palsy resolved after 2 weeks, but unfortunately his right-sided hemiparesis persisted necessitating long-term care placement.

Conjugate gaze deviation is relatively common in ischemic and hemorrhagic stroke and is found in approximately 15\%-30\%

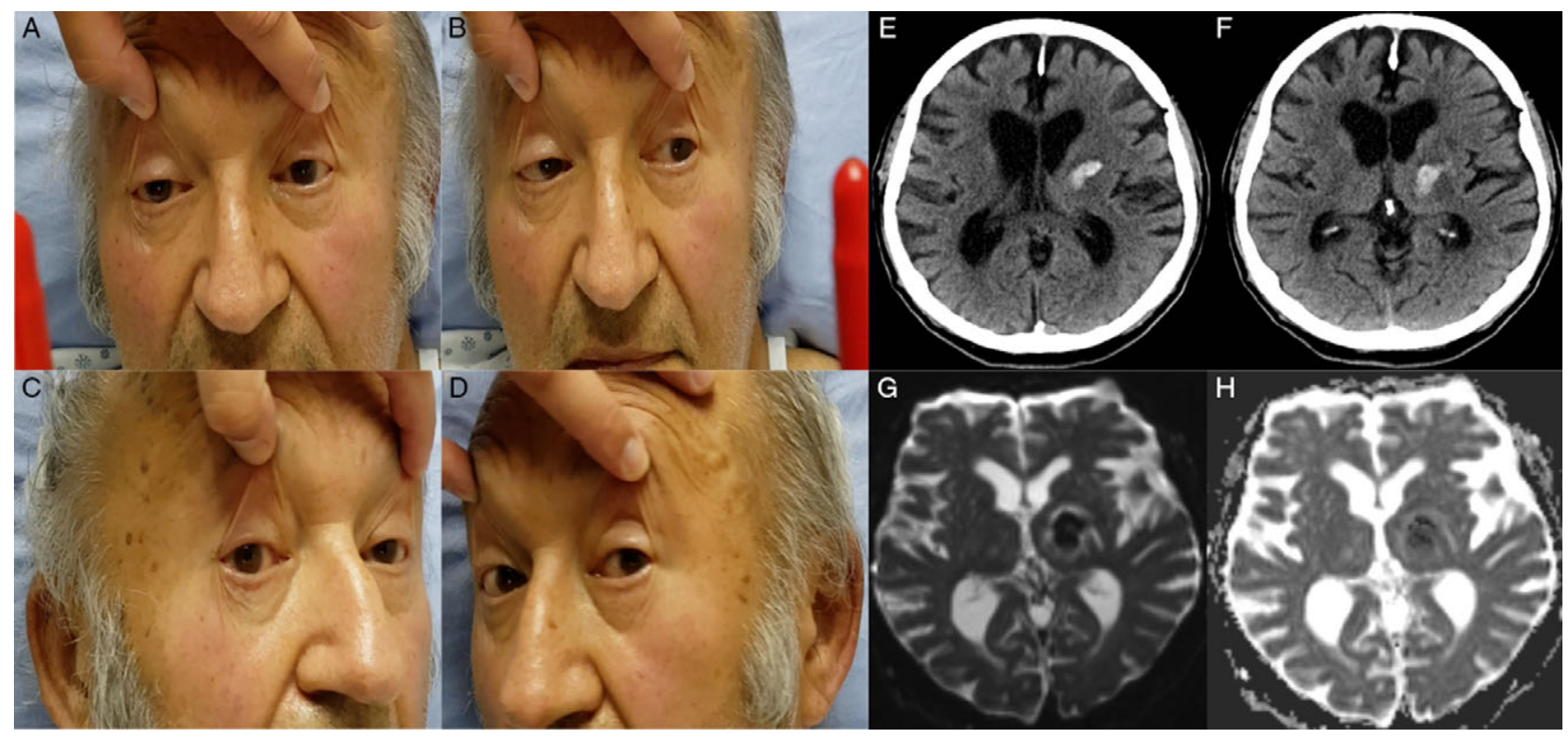

Figure 1: Supranuclear rightward gaze palsy from left anterior internal capsule hemorrhage. On examination of extraocular movements, the patient had impaired rightward (A) but preserved leftward (B) gaze on evaluation of smooth pursuit and voluntary horizontal saccades. With the oculocephalic maneuver, there is improvement in the rightward gaze palsy $(C)$ with preservation of the leftward response $(D)$. (E, $F)$ Noncontrast enhanced CT axial images demonstrate a hyperdense lesion with surrounding hypodensity representing acute hemorrhage and associated edema within the left posterior limb of the internal capsule, extending into the anterior internal capsule. Day 2 diffusion weighted $(G)$ and apparent-diffusion coefficient $(H)$ MRI sequences both demonstrate an area of decreased signal and surrounding increased signal consistent with acute hemorrhage and surrounding edema within the left anterior and posterior internal capsule and medial thalamus. No additional lesions were identified through these modalities to otherwise explain the clinical presentation with supranuclear rightward gaze palsy.

\footnotetext{
From the Department of Medicine (Neurology), University of Alberta, Edmonton, Alberta, Canada (NC, GB, MWH)

Received March 29, 2020. Final Revisions Submitted May 22, 2020. Date of Acceptance May 30, 2020.

Correspondence to: Dr. M. Wasif Hussain, 7-132 Clinical Sciences Building, 11350-83 Ave, Edmonton, AB T6G 2G3, Canada. Email: mhussain@ualberta.ca
} 


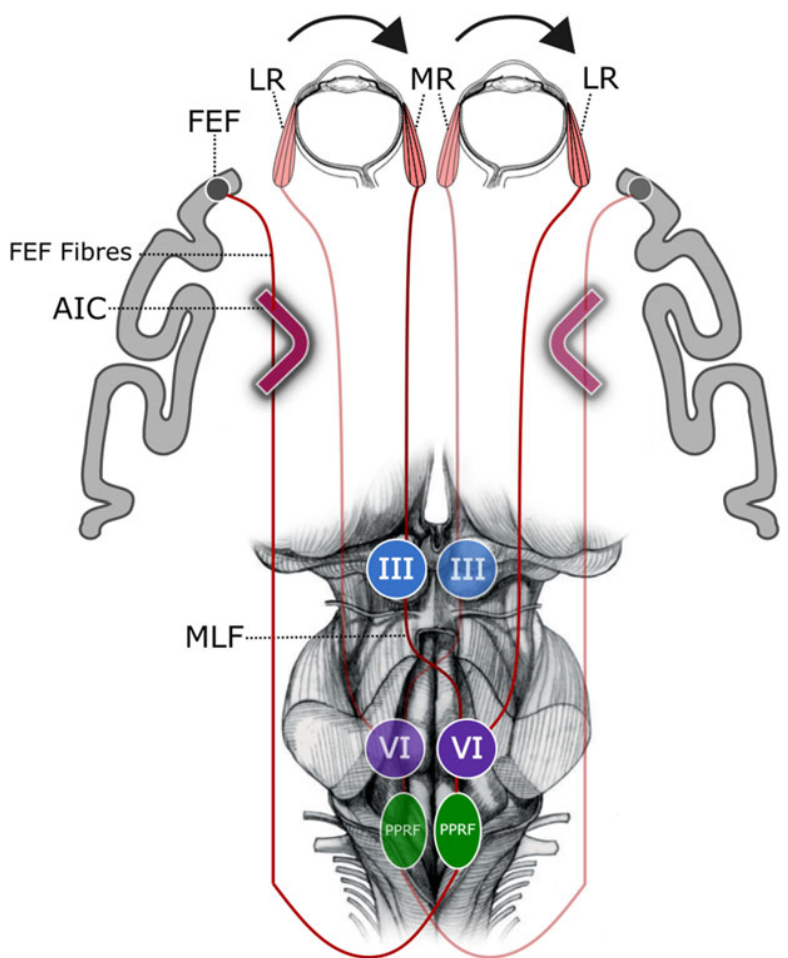

Figure 2: Neuroanatomy of voluntary conjugate horizontal saccades. Generation of voluntary conjugate horizontal saccades is initiated via impulses from the cortical frontal eye fields $(F E F)$ which send projections through the anterior internal capsule (AIC). The FEF fibers descend to the contralateral paramedian pontine reticular formation $(P P R F)$ which in turn sends impulses to the ipsilateral abducens (VI) nucleus. The VI nucleus sends fibers to the contralateral oculomotor (III) nucleus via the medial longitudinal fasciculus $(M L F)$. Cranial nerves III and VI then activate the contralateral medial rectus $(M R)$ and ipsilateral lateral rectus (LR) muscles, respectively, to generate a saccade contralateral to the initially activated FEF. Adapted from Neuroanatomy Through Clinical Cases by H. Blumenfeld, 2010, Sinauer Associates. Copyright 2018 by Oxford University Press. Adapted with permission.

of cases. ${ }^{1,2}$ Anatomically, the frontal eye fields (FEF) located between the premotor and prefrontal cortex are crucial for supranuclear control of horizontal conjugate gaze. ${ }^{3}$ Descending FEF fibers project to the paramedian pontine reticular formation (PPRF) in the brainstem, and lesions of this circuit (Figure 2) at the PPRF, abducens nucleus, and/or the medial longitudinal fasciculus produce various horizontal oculomotor deficits, including total conjugate lateral gaze palsy, unilateral or bilateral internuclear ophthalmoplegia, and one-and-a-half syndrome. ${ }^{3}$

The majority of stroke cases with conjugate gaze deviation are seen with cortical lesions affecting the FEF, as opposed to our case with a lesion of the anterior limb of the internal capsule. The internal capsule possesses highly conserved topography, with the genu containing the corticobulbar tract, and the posterior limb carrying corticospinal, corticorubral, and corticopontine tracts in addition to ascending sensory thalamocortical fibers. ${ }^{4}$ The anterior limb of the internal capsule contains predominantly frontopontine tracts and thalamocortical fibers. ${ }^{5}$

Our case highlights an uncommon cause of horizontal supranuclear gaze palsy from disruption of FEF fibers in the anterior limb of the internal capsule. The intact vestibulo-ocular reflex and relatively rapid resolution of the gaze palsy in our case is more consistent clinically with a FEF supranuclear lesion, compared with a pontine lesion, and neuroimaging did not identify a pontine lesion to otherwise explain the patient's deficits. From a neuroanatomical perspective, descending FEF fiber bundles are transmitted via the anterior limb of the internal capsule, and have been experimentally demonstrated using anterograde tracers injected into the FEF. ${ }^{6,7}$ Thus, a lesion such as a hemorrhage or mass in the anterior limb of the internal capsule may cause contralateral conjugate gaze palsy. This appears to be under-reported in the literature, with only one previously published case report of a contralateral saccadic gaze palsy secondary to a hematoma in the internal capsule. ${ }^{8}$ This is surprising, given that the majority $(>80 \%)$ of spontaneous intracerebral hemorrhages occur in deep parenchymal regions such as thalamus, basal ganglia, or the internal capsule. ${ }^{9}$

\section{Disclosures}

$\mathrm{NC}, \mathrm{GB}$, and MWH have no disclosures to report.

\section{Statement of Authorship}

$\mathrm{NC}$ drafted the manuscript, created the figures, and wrote the corresponding legends. GB performed the patient's clinical assessment, and edited the manuscript. MWH assisted in the patient's clinical assessment, and performed a critical revision of the manuscript for intellectual content.

\section{REFERENCES}

1. De Renzi E, Colombo A, Faglioni P, Gibertoni M. Conjugate gaze paresis in stroke patients with unilateral damage. An unexpected instance of hemispheric asymmetry. Arch Neurol. 1982;39(8):482-6. doi: 10.1001/archneur.1982. 00510200024004

2. Mohr J, Rubinstein LV, Kase C. Gaze palsy in hemispheral stroke: the NINCDS stroke data bank. Neurology. 1984;34(Suppl 1):199.

3. Blumenfeld H. Neuroanatomy through clinical cases, 2nd ed. Sunderland: Sinauer Associates; 2010.

4. Emos MC, Agarwal S. Neuroanatomy, internal capsule. [Updated June 3, 2019]. In: StatPearls [Internet]. Treasure Island, FL: StatPearls Publishing; 2020. Available at: https:// www.ncbi.nlm.nih.gov/books/NBK542181/; accessed May 23, 2020.

5. Mithani K, Davison B, Meng Y, Lipsman N. The anterior limb of the internal capsule: anatomy, function, and dysfunction. Behav Brain Res. 2020;387:112588. doi: 10.1016/j.bbr.2020.112588

6. Brazis PW, Biller J, Masdeu JC. Localization in clinical neurology, 6th ed. Philadelphia: Wolters Kluwer/Lippincott Williams \& Wilkins Health; 2011.

7. Stanton GB, Goldberg ME, Bruce CJ. Frontal eye field efferents in the macaque monkey: I. Subcortical pathways and topography of striatal and thalamic terminal fields. J Comp Neurol. 1988;271(4):473-92.

8. Fukutake T, Hirayama K, Sakakibara R. Contralateral selective saccadic palsy after a small haematoma in the corona radiata adjacent to the genu of the internal capsule. J Neurol Neurosurg Psychiatry. 1993;56(2):221.

9. Qureshi AI, Palesch YY, Barsan WG, et al. Intensive blood-pressure lowering in patients with acute cerebral hemorrhage. N Engl J Med. 2016;375(11):1033-43. 